\title{
Scoping the future for interventional radiology and interventional neuroradiology: a clinical physics perspective
}

\author{
Zoe Brady $^{1,2} \cdot \operatorname{lan}$ Smith $^{3} \cdot$ Nicholas Brown $^{4,5}$
}

Accepted: 22 February 2022 / Published online: 7 March 2022

๑) Australasian College of Physical Scientists and Engineers in Medicine 2022

\section{Background}

Interventional Radiology (IR) and Interventional Neuroradiology (INR) are complementary specialties within clinical radiology that are relative newcomers to modern medicine. The first interventional radiology procedure was performed in 1964 by pioneering radiologist Charles Dotter, which heralded the beginning of image-guided and endovascular specialties. The Royal Australian and New Zealand College of Radiologists (RANZCR) has recently developed a framework [1] to achieve formal specialty recognition for IR and INR through the Australian Medical Council (AMC) and Australian Health Practitioner Regulation Agency (AHPRA) processes.

IR and INR disciplines are collectively small, but very dynamic and rapidly evolving, specialties that provide critical services to patients and hospitals. The range of practice for IR and INR involve image-guided endovascular, endoluminal and percutaneous treatment of patients. IR procedures focus on peripheral and central vasculature, hollow viscera and solid organs, the musculoskeletal system including vertebral column and the peripheral nervous system. INR practice specialises in treating diseases of the neurovasculature, brain, sensory organs, meninges, cerebrospinal fluid (CSF), head and neck, spinal cord, vertebral column and the peripheral nervous system. These specialist diagnostic radiologists utilise image-guidance across the range of imaging

Zoe Brady

z.brady@alfred.org.au

1 Department of Radiology, Alfred Health, Melbourne, Australia

2 Department of Neuroscience, Monash University, Melbourne, Australia

3 Physical Sciences \& Clinical Outcomes Analysis, St Andrew's War Memorial Hospital, Brisbane, Australia

4 The Wesley Hospital, Brisbane, Australia

5 The University of Queensland, Brisbane, Australia modalities, including fluoroscopy/angiography, Computed Tomography (CT), ultrasound (US), and increasingly Magnetic Resonance Imaging (MRI).

In addition to interventional radiologists and neuroradiologists, a number of other specialists also have a role in delivery of the endovascular angiographic subset of interventional procedures. These include vascular surgeons and cardiologists, while neurologists, neurosurgeons, thoracic surgeons and renal physicians are all specialties with a small percentage of people working in a space that overlaps the IR and INR fields. Over the last 5-10 years these groups have increasingly become more accustomed to working with each other, sharing patient care to improve patient outcomes.

Given the RANZCR road map1 to formal professional recognition and the role of advanced technology and applications in the specialty, a "future scoping" session was held at the 2021 Engineering in Physical Sciences and Medicine (EPSM) Conference. A dialogue between a clinical medical physicist (ZB) and an interventional radiologist (NB) was used to stimulate discussion and forecast ideas on where the discipline is heading with a view to the role of a medical physicist. Live polls were put to attendees from a medical physics and/or engineering background where most (if not all) have experience in supporting medical imaging or interventional services $(n=17)$, with discussion focussed on advanced IR and INR procedures and horizon scanning from a medical physics perspective.

\section{Technology}

High-end technology is a critical component of IR and INR, with modern angiography suites becoming increasingly complex, commonly utilising advanced vessel navigation software, cone-beam CT and bi-plane flat panel detector capabilities. Attendees were asked to rate which technologies, in their opinion, would have the most significant impact in the IR field in the future. Figure 1 suggests that robotics 


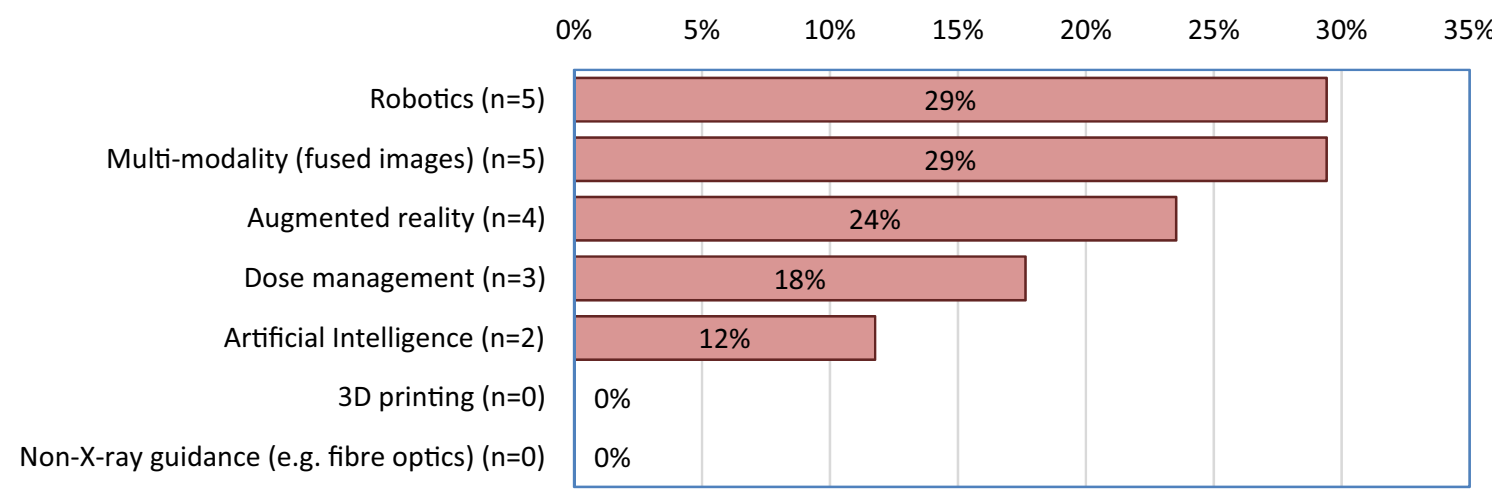

Fig. 1 Attendee $(n=17)$ live poll response to the question "What new technology will have a significant impact in the IR field?". Attendees could select multiple answers

and fusing images through multiple modalities were rated equally highly, followed closely by augmented reality applications. Interestingly, despite being "on trend" and an active focus of current research, Artificial Intelligence (AI) was not ranked as significant in comparison by those participating in the meeting. Non-X-ray guidance (e.g. fibre optics) and 3D printing failed to score any votes in the poll.

It is likely that clinical implementation of robotics in IR and INR will become more common within the next decade. Robotic technology offers a number of potential efficiencies and gains in clinical practice, including radiation dose reduction, greater control of techniques requiring fine movements, and semi-automation of some processes. The next phase may enable cases to be performed remotely, which would not only reduce operator radiation dose but could dramatically improve service delivery and patient access to specialty treatment that would otherwise have been unlikely. However, significant challenges do exist, chiefly high costs that are prohibitive for many centres and an equivocal cost-benefit profile. Interventional radiologists and neuroradiologists also greatly rely on tactile senses, which can be difficult to replicate with a robotic device. Whilst regularly having robots in labs locally, with operators positioned outside of the angiography suite, is still some time away, the relentless evolution of technology promises to deliver this reality within the next decade.

Early precursors of image fusion exist with the overlay of 3D images (e.g. from rotational angiography) on 2D images, which has assisted interventional radiologists navigate through arteries and triangulate targets for ablative or embolic treatments. Similarly, image-fusion using a combination of modalities including MRI, positron emission tomography (PET) scans and US are already widely used.

As with other technological advancements, AI has the potential to raise quality standards, increase workload and improve diagnostic accuracy through the ability to extract information from big data. For example, AI may be able to specify the best catheter based on a patient's specific anatomy, or optimise localisation of target lesions for treatment. It may also enable better identification of patients likely to benefit from different interventions, or improve selection of treatment options to minimise unnecessary procedures and episodes of low-value care. The promises of AI are imminent, with augmented applications becoming increasingly visible in this field over the next 3-5 years.

\section{Clinical Applications}

The expansion of IR and INR is being driven by research that demonstrates improved long-term patient outcomes from image-guided treatment options in certain subsets of patients. For example, the treatment of some cancers previously involved major surgeries with significant risk factors and co-morbidities and long-term recovery rates. Minimally invasive radiological procedures now offer potentially curative treatment with the same or similar outcomes as surgery, with lower complication rates, reduced treatment costs and faster recovery times. Using IR techniques alongside existing surgical techniques and medical treatments will optimise therapeutic options and maximise high-value care. It also allows for a reallocation of resources to where they will be most effective.

The current and future applications of IR are increasingly relevant to oncology, leading to the emergence of interventional oncology (IO) as an important field over the last 10-15 years. Internationally, IO is recognised as the fourth pillar of oncology care alongside surgery, medical oncology and radiation oncology. IR can now utilise interventional techniques to treat tumours with curative or palliative intent in just about every organ of the body. For example, liver directed treatments using both chemoembolisation and radioembolisation as well as percutaneous ablative techniques 
are becoming more prevalent. Some of these techniques may also be developed for cancers in other organs as well.

In the INR space over the last 10 years there has been a rapid shift towards endovascular treatments for stroke patients. Mechanical thrombectomy is a potentially lifechanging or life-saving technique and has become standard of care for many stroke patients. Neuroradiologists retrieve clots from blocked arteries in the brain that can completely change the outlook for these patients. INR will also have a greater role in the treatment of non-neurological, neurological and cerebral tumours. Expertise and evidence gained in the IR and IO space can be leveraged to expand treatment options for brain cancers and other central nervous systems tumours.

\section{Medical Physics}

Historically in Australia, clinical radiologists have had the most contact with medical physicists when training for their physics (applied imaging technology) exams during registrar training. As a result of few diagnostic imaging physicists in practice and the absence (almost entirely) of medical physicists in private practice, there is often little exposure to this vital area. Often the most visible role for medical physicists is within the field of radiation safety in terms of personal protective equipment advice and compliance (radiation badges, lead glass protective eyewear, radiation protective aprons, in-room protective devices).
More than $50 \%$ of attendees at the conference session felt that medical physicists are currently equipped to acceptance test new technologies in the clinical environment, manage and/or perform routine quality control and participate in the development and running of continuous quality monitoring (CQM) processes hence contributing to overall quality improvement (Fig. 2). Attendees responded that they were least equipped to actively participate as members of a multidisciplinary team in horizon scanning, which was interesting given the subject of the conference session.

A recommended method for greater involvement in the clinical environment by medical physicists is to attend multidisciplinary team (MDT) meetings. These meetings traditionally focus on patient care and are becoming increasingly common within hospitals. The details around a patient case are presented for review by colleagues from multiple specialties (i.e. the users of radiology) and to seek advice on the next course of action (e.g. treatment, further investigations, more diagnosis). This can be valuable in determining appropriate patient management. In the MDT environment, contributions from other fields that provide alternative perspectives, can be valuable. Being present at these meetings will allow medical physicists to develop a profile and reputation for the services they provide to assist in optimising patient care. There is direct benefit to all professional groups from gaining an understanding of what others do in their role and hence determining how a medical physicist's skillset can be best utilised [2]. Increasing exposure to and involvement with the clinical team will also create opportunities

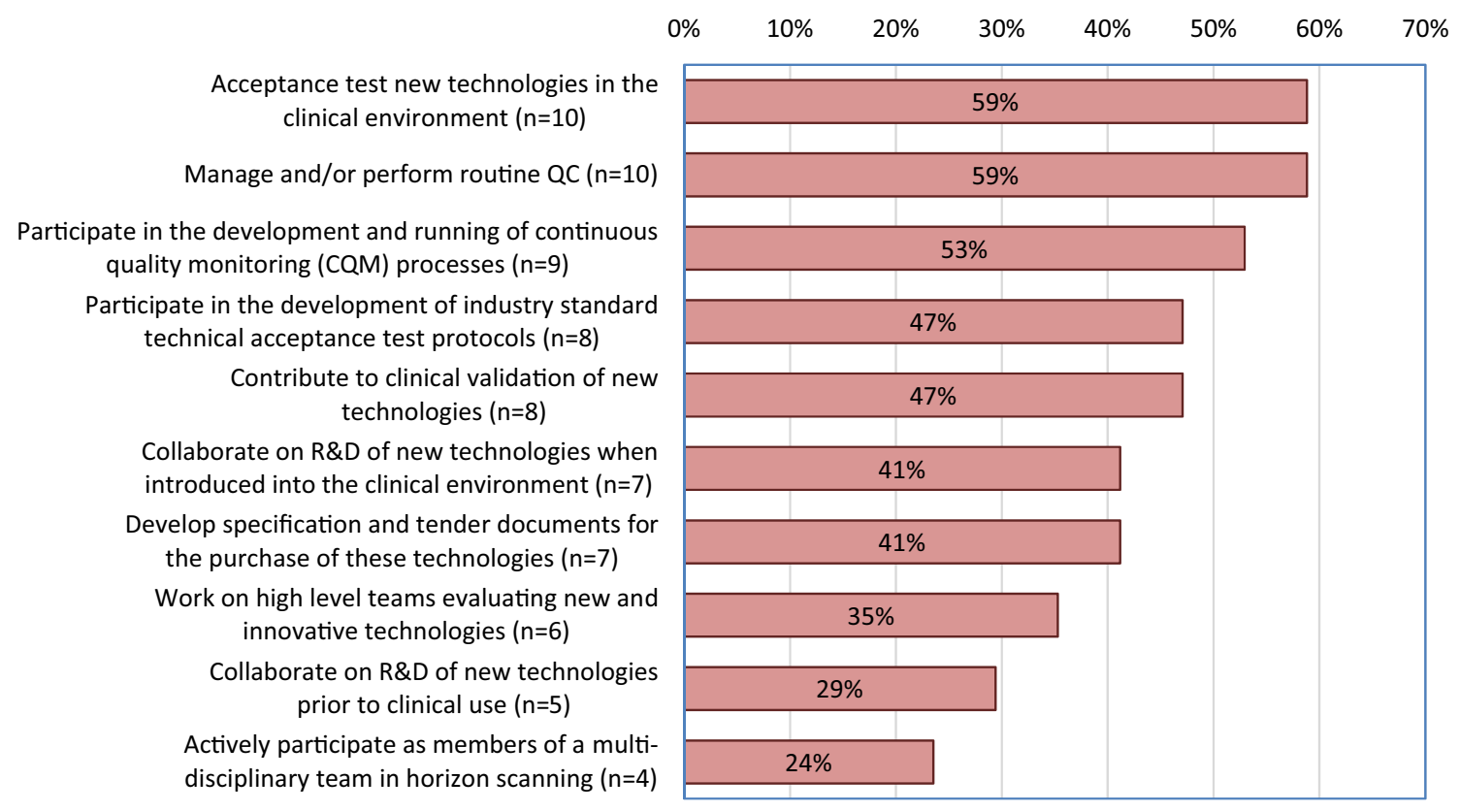

Fig. 2 Attendee $(n=17)$ live poll response to the question "Which of the following roles do you believe medical physicists are currently equipped to participate in?". Attendees could select multiple answers 
for research collaboration, which is an important aspect of new technology development, validation, optimisation and implementation.

\section{Conclusion}

In conclusion, disruptive technologies will continue to be developed and evolve the way IR and INR work is performed. Changes that improve treatment efficacy, shorten the duration of care, reduce costs and allow better patientcentred outcomes are highly desirable. Furthermore, new technical capabilities will allow scope of practice to alter and possibly expand, as has been experienced within interventional oncology and endovascular treatment of stroke. An optimal patient-centric system will make use of all experts that are available in the clinical workplace. For medical physicists, this may mean taking the first step in approaching an interventional radiologist or neuroradiologist. Ultimately, embracing these changes by working together in multidisciplinary teams will result in the best outcomes for the patient.

\section{References}

1. The Royal Australian and New Zealand College of Radiologists (RANZCR), The faculty of clinical radiology, a road map for advancing interventional radiology and interventional neuroradiology in Australia and New Zealand, Version 1.0 (2020) www. ranzcr.com, accessed October 2021

2. The Australasian College of Physical Scientists \& Engineers in Medicine (ACPSEM) Position statement, the role of physicists, scientists and engineers in medicine in Australasia (2018) www. acpsem.org.au, accessed February 2022

Publisher's Note Springer Nature remains neutral with regard to jurisdictional claims in published maps and institutional affiliations. 